\title{
Patterns of Oscillation in a Ring of Identical Cells with Delayed Coupling
}

\author{
Sharene D. Bungay ${ }^{* \dagger} \quad$ Sue Ann Campbell ${ }^{\dagger}$
}

July 26, 2006

\begin{abstract}
We investigate the behaviour of a neural network model consisting of three neurons with delayed self and nearest-neighbour connections. We give analytical results on the existence, stability and bifurcation of nontrivial equilibria of the system. We show the existence of codimension two bifurcation points involving both standard and $D_{3}$-equivariant, Hopf and pitchfork bifurcation points. We use numerical simulation and numerical bifurcation analysis to investigate the dynamics near the pitchfork-Hopf interaction points. Our numerical investigations reveal that multiple secondary Hopf bifurcations and pitchfork bifurcations of limit cycles may emanate from the pitchforkHopf points. Further, these secondary bifurcations give rise to 10 different types of periodic solutions. In addition, the secondary bifurcations can lead to multistability between equilibrium points and periodic solutions in some regions of parameter space. We conclude by generalizing our results into conjectures about the secondary bifurcations that emanate from codimension two pitchfork-Hopf bifurcation points in systems with $D_{n}$ symmetry.
\end{abstract}

\section{Introduction}

The work of Golubitsky et al. [1988] shows that systems with symmetry can lead to many interesting patterns of oscillation, which are predictable based on the theory of equivariant bifurcations. In a series of papers Wu, et al. [Krawcewicz et al., 1997; Krawcewicz and Wu, 1999; Wu, 1998] extended the theory of equivariant Hopf bifurcation to systems with time delays (functional differential equations). It should be noted that this theory predicts the

\footnotetext{
${ }^{*}$ Current address: Department of Computer Science, Memorial University of Newfoundland, St. John's, Newfoundland and Labrador, A1B 3X5, Canada.

${ }^{\dagger}$ Department of Applied Mathematics, University of Waterloo, Waterloo, Ontario, N2L 3G1, Canada.
} 
possible patterns of oscillation in a system solely on the symmetry structure of the system. To understand which patterns occur in a particular system and whether they are stable, one needs to consider a specific model for a system.

With this in mind, there has been interest in applying these results to models related to the Hopfield-Cohen-Grossberg neural networks [Cohen and Grossberg, 1983; Grossberg, 1978, 1980; Hopfield, 1984, 1982] with time delays [Marcus et al., 1991; Marcus and Westervelt, 1989]. Such models make an ideal test bed for this theory as the models for the individual elements are quite simple (one variable for each element), yet with the introduction of time delays the behaviour can be quite complex. The focus of this work has been on networks with a ring structure with nearest-neighbour (bi-directional) coupling between the elements. This leads to a system with $\mathbf{D}_{n}$ symmetry, i.e. a system which has the symmetries of a polygon with $n$ sides of equal length. Most of these studies have concerned lower dimensional systems (e.g [Campbell et al., 2006; Guo et al., 2004; Ncube et al., 2003; Shayer and Campbell, 2000; Wu et al., 1999]) and/or systems with a single time delay [Guo, 2005; Guo and Huang, 2003, 2005, 2006; Wu, 1998; Wu et al., 1999]. In previous work [Yuan and Campbell, 2004; Campbell et al., 2005] we studied the stability and bifurcations (both standard and equivariant) of the trivial solution for a ring of arbitrary size with two time delays. There is also work on Hopfield-Cohen-Grossberg networks with a ring structure and uni-directional coupling [Baldi and Atiya, 1994; Campbell, 1999; Campbell et al., 1999].

Other work on systems with symmetry and time delays includes that of Orosz and Stépán [2004], who studied a quite general system with translational symmetry and one time delay using centre manifold and normal form analysis. In this and a subsequent paper [Orosz and Stépán, 2006], they applied their results to a car following model with periodic boundary conditions, which leads to a system with a ring structure and uni-directional coupling. Orosz et al. [2004, 2005] studied this model further using local bifurcation theory and numerical continuation analysis.

This paper investigates the behaviour of a 3-dimensional bi-directional neural network model with delayed self and nearest-neighbour connections, as shown in Fig. 1. The strength 


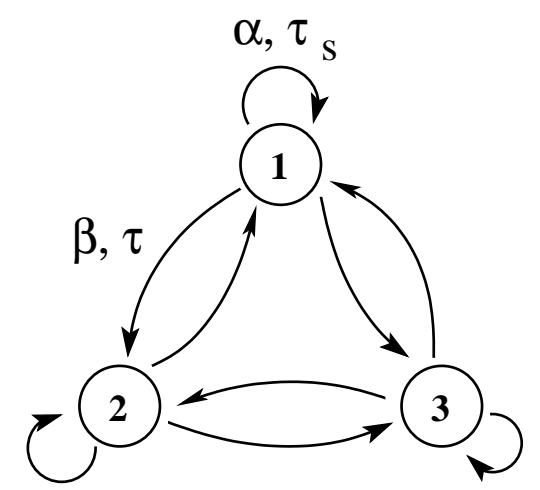

Figure 1: Ring of three neurons with delayed coupling and self feedback.

of the self and nearest-neighbour coupling is denoted by $\alpha$ and $\beta$, respectively, while $\tau_{s}$ and $\tau$ denote the corresponding delays.

The following model is used to simulate the ring of three neurons shown in Fig. 1:

$$
\dot{x}_{i}(t)=-x_{i}(t)+\alpha \tanh \left(x_{i}\left(t-\tau_{s}\right)\right)+\beta\left[\tanh \left(x_{i-1}(t-\tau)\right)+\tanh \left(x_{i+1}(t-\tau)\right)\right],
$$

where $i \bmod 3$. The individual elements are represented by a scalar equation, consisting of a linear decay term and a nonlinear, time delayed self connection (feedback). Here $\tau>0$ represents the time delay in the connections between different elements and $\tau_{s}>0$ the time delay in the self connection. The sign of the coupling coefficients $\alpha$ and $\beta$ indicate whether the connection is excitatory (positive) or inhibitory (negative).

A complete description of the linear stability of the trivial solution of (1) was given in Campbell et al. [2006]. It was noted that the trivial solution may lose stability through one of four bifurcations: standard or equivariant pitchfork bifurcations and standard or equivariant Hopf bifurcation. Fig. 2 shows a two parameter representation of the bifurcations exhibited by (1). The standard Hopf bifurcation leads to synchronous oscillations, i.e. oscillations where all the components oscillate in-phase. The equivariant Hopf bifurcation leads to eight branches of asynchronous oscillations of three types: (i) phase-locked oscillations, where the three components oscillate with the same wave pattern but one-third period out of phase with each other; (ii) standing waves, where two components oscillate with the same wave pattern but half a period out of phase with each other while the third is fixed at zero; and 


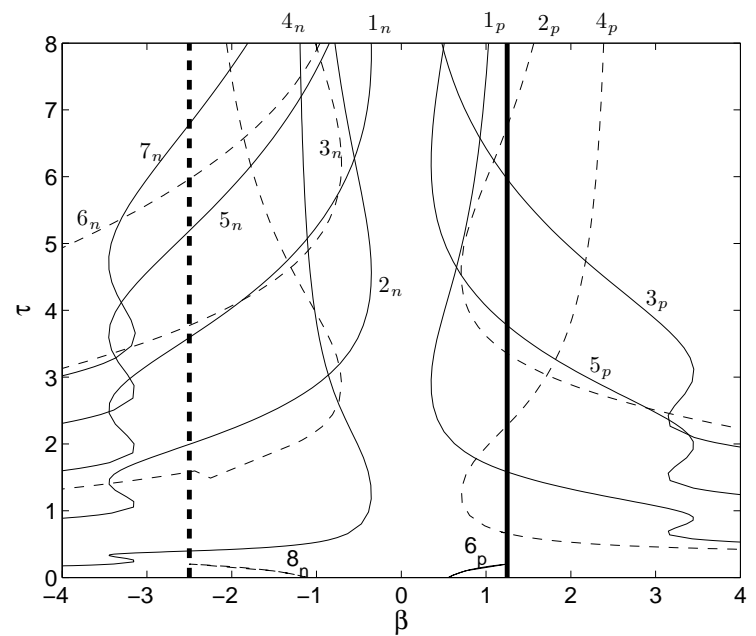

Figure 2: Bifurcation curves of the model (1) in the $\beta-\tau$ parameter space. The other parameter values are: $\alpha=-1.5, \tau_{s}=1$. Solid lines represent standard bifurcations, dashed lines represent equivariant bifurcations. Thin lines represent Hopf bifurcations, while thick lines represent steady state (pitchfork) bifurcations.

(iii) mirror reflecting waves, where two components oscillate in phase with each other while the third component oscillates half a period out of phase with them and with twice the amplitude. An example of each of these asynchronous oscillations is shown in Fig. 3.

Centre manifold analysis was used to determine the local stability of the synchronous oscillations in Ncube et al. [2003]; Yuan and Campbell [2004] and of all branches of asynchronous oscillations in Campbell et al. [2005]. Perturbation analysis was used to determine the local stability of the phase-locked oscillations in Campbell et al. [2006].

It has been noted [Bélair and Campbell, 1994; Campbell, 1999] that systems with multiple time delays can often have codimension two bifurcation points, also called bifurcation interaction points. This is true of system (1) as well [Campbell et al., 2006, 2005]. Three common types of codimension two bifurcation points are points in parameter space where the characteristic equation has a double zero root, a zero root and a pair of pure imaginary roots, or two pairs of pure imaginary roots. These points are referred to, respectively, as a Bogdanov bifurcation point, a steady state-Hopf interaction point and a Hopf-Hopf interaction point. Generically, such points can be found only in systems with at least two parame- 


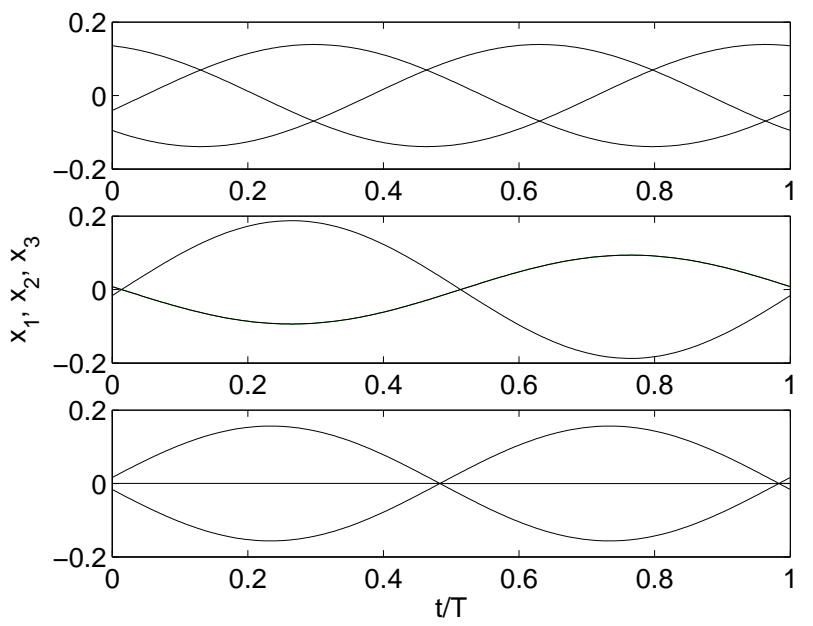

Figure 3: An illustration of the types of oscillations that can arise as a result of an equivariant Hopf bifurcation in the model (1). From top to bottom we have: Phase-locked oscillations, mirror-reflecting oscillations, and standing-wave oscillations.

ters. In a two parameter representation of the bifurcations curves of a system, codimension two bifurcation points occur at the intersection points of two different bifurcation curves. The behaviour of systems near each of the codimension two bifurcation points listed above has been completely investigated, using singularity theory, for standard Hopf and standard steady state bifurcations. Various curves of secondary bifurcations are shown to emanate from these points. A complete review of these results can be found in Guckenheimer and Holmes [1983, Chapter 7] or Kuznetsov [1995, Chapter 8].

By contrast, very little theory exists for codimension two bifurcations involving equivariant bifurcations. One way to investigate such points is using numerical bifurcation analysis, also called numerical continuation. Numerical continuation software uses an iterative procedure to approximate equilibrium points and periodic solutions of a differential equation. By repeating this procedure for many parameter values, the program produces a bifurcation diagram: a plot of some representation of the norm of each equilibrium point and periodic solution as a function of a parameter. The curves in such a diagram are usually referred to as branches of solutions. The stability of each solution and how it changes along the 
branch is calculated and hence bifurcations points can be located. Most numerical continuation programs also have the capability to continue the bifurcation points to produce two parameter plots of bifurcation curves. Some commonly used numerical continuation packages are AUTO [Doedel et al., 1991a,b], and MatCont [Dhooge et al., 2003], however, these only deal with ordinary differential equations and iterated maps. The package DDEBIFTOOL [Engelborghs et al., 2001] performs numerical continuation for delay differential equations.

Consideration of the many intersection points of bifurcation curves in Fig. 2 shows that our system exhibits bifurcation interactions involving standard bifurcations, equivariant bifurcations and mixed interactions involving standard and equivariant bifurcations. It is the purpose of this paper to investigate these points numerically, using the bifurcation continuation software DDE-BIFTOOL and the numerical simulation tool XPPAUT [Ermentrout, 2002]. The goal of our work is twofold: (i) to shed light on the possible oscillation patterns which can occur in the network of Fig. 1; and (ii) to make conjectures as to the types of secondary bifurcations that can arise from codimension two points involving equivariant bifurcations. The focus of the paper will be on steady state-Hopf interaction points.

The outline of the paper is as follows. In Sec. 2 we will give some analytical results on the existence of nontrivial equilibria and of their Hopf bifurcation. In Sec. 3 we will present our numerical results. In Sec. 4 we will summarize our results and draw some conclusions.

\section{Existence and Bifurcation of Nontrivial Equilibria}

In addition to the trivial solution, Eqs. (1) admit various types of nontrivial equilibria. We begin by determining the steady state bifurcations of the trivial solution which may lead to the creation of nontrivial equilibria. We then describe the possible types of nontrivial equilibria and give constraints on the parameters for them to occur. This will then lead to some conclusions about the type and criticality of the steady state bifurcations that occur. Finally, by considering the characteristic equation of the linearization of (1) about each of these nontrivial equilibria, we will draw conclusions about the kinds of oscillations that can 
be produced by Hopf bifurcations of these equilibria.

To study the steady state bifurcations of the trivial solution of (1), one must consider the the characteristic equation of the linearization of (1) about the trivial solution. In Campbell et al. [2006, 2005] it was shown that this is given by

$$
\left(-1-\lambda+\alpha e^{-\tau_{s} \lambda}+2 \beta e^{-\tau \lambda}\right)\left(-1-\lambda+\alpha e^{-\tau_{s} \lambda}-\beta e^{-\tau \lambda}\right)^{2}=0
$$

Clearly the characteristic equation has a simple zero root when $-1+\alpha+2 \beta=0$ and a double zero root when $-1+\alpha-\beta=0$. Thus the former is a potential standard steady state bifurcation point, while the latter is a potential equivariant steady state bifurcation point. Since the nonlinearities in the model (1) are odd functions these will be pitchfork bifurcations [Guckenheimer and Holmes, 1983, Section 3.4].

The simplest nontrivial equilibria are of the form $\left(x^{*}, x^{*}, x^{*}\right)$ where $x^{*}$ satisfies

$$
-x^{*}+\alpha \tanh \left(x^{*}\right)+2 \beta \tanh \left(x^{*}\right)=0
$$

We call these nontrivial synchronous equilibria since all three of the components are the same.

Theorem 1: If $\alpha+2 \beta>1$ then there are exactly two nontrivial synchronous equilibria $\left(x^{*}, x^{*}, x^{*}\right)$ and $\left(-x^{*},-x^{*},-x^{*}\right)$. If $\alpha+2 \beta<1$, there are no nontrivial synchronous equilibria.

Proof: Let $F(x)=-x+(\alpha+2 \beta) \tanh (x)$. Note that $F$ is odd (hence $F(0)=0), F^{\prime}(x)=$ $-1+(\alpha+2 \beta) \operatorname{sech}^{2}(x)$ and $F^{\prime \prime}(x)=-2(\alpha+2 \beta) \operatorname{sech}^{2}(x) \tanh (x)$.

Let $\alpha+2 \beta<1$. Then $(\alpha+2 \beta) \operatorname{sech}^{2}(x)<\operatorname{sech}^{2}(x) \leq 1$, i.e. $-1+(\alpha+2 \beta) \operatorname{sech}^{2}(x)<0$. So $F$ is strictly decreasing for all $x$ and there can be at most one $x$ such that $F(x)=0$. Since $F(0)=0$, there are no nontrivial equilibria.

Let $\alpha+2 \beta>1$. Then $F^{\prime}(0)>0$ and $\lim _{x \rightarrow \infty} F^{\prime}(x)<0$ thus there is an $\bar{x}>0$ such that $F^{\prime}(\bar{x})=0$. Now $F^{\prime \prime}(x)<0$ for $x>0$, thus there is only one such $\bar{x}$. Thus we have $F^{\prime}(x)>0$ for $0 \leq x<\bar{x}$ and $F^{\prime}(x)<0$ for $x>\bar{x}$. It follows that there is exactly one $x^{*}>\bar{x}$ such that $F\left(x^{*}\right)=0$. By symmetry $F\left(-x^{*}\right)=0$. 
This result about the existence of synchronous equilibria allows us to make a conclusion about bifurcation of the trivial solution.

Theorem 2: There is a supercritical pitchfork bifurcation of the trivial solution of system (1) at $\alpha+2 \beta=1$, leading to two branches of synchronous equilibria given by $\left( \pm x^{*}, \pm x^{*}, \pm x^{*}\right)$ where $x^{*}$ satisfies (3).

Proof: It is clear from the characteristic equation (2) that the trivial solution gains a real eigenvalue with positive real part as $\alpha+2 \beta$ increases through 1 . From the previous theorem, the synchronous equilibria $\left( \pm x^{*}, \pm x^{*}, \pm x^{*}\right)$ exist only for $\alpha+2 \beta>1$. Finally, it can be shown that $x^{*} \rightarrow 0$ as $\alpha+2 \beta \rightarrow 1^{+}$. The result follows.

By analogy with the oscillations which arise from the equivariant Hopf bifurcation of the trivial solution, we define two types of asynchronous equilibria: standing wave equilibria and mirror reflecting equilibria. Symmetry arguments show that these are the only types of asynchronous equilibria which bifurcate from the trivial solution in a system with $D_{3}$ symmetry [Golubitsky et al., 1988, Chapter XV §4].

Standing wave equilibria are of the form $\left( \pm x^{*}, \mp x^{*}, 0\right)$ (and permutations thereof) where $x^{*}$ satisfies

$$
-x^{*}+\alpha \tanh \left(x^{*}\right)+\beta \tanh \left(-x^{*}\right)=0
$$

Theorem 3: If $\alpha-\beta>1$ then there are exactly six nontrivial standing wave equilibria: $\left( \pm x^{*}, \mp x^{*}, 0\right)$ and their permutations, where $x^{*}$ is given by Eq.(4). If $\alpha-\beta<1$, there are no nontrivial standing wave equilibria.

Proof: Let $F(x)=-x+(\alpha-\beta) \tanh (x)$. The proof is essentially the same as for the synchronous equilibria.

Mirror reflecting equilibria are of the form $\left( \pm x^{*}, \pm x^{*}, \pm y^{*}\right)$ (and permutations thereof) where $x^{*}, y^{*}$ satisfy

$$
\begin{array}{r}
-x^{*}+\alpha \tanh \left(x^{*}\right)+\beta \tanh \left(x^{*}\right)+\beta \tanh \left(y^{*}\right)=0 \\
-y^{*}+\alpha \tanh \left(y^{*}\right)+2 \beta \tanh \left(x^{*}\right)=0
\end{array}
$$


Theorem 4: If $\alpha<1$ and $\alpha-\beta>1$ then there are at least six nontrivial mirror reflecting equilibria: $\left( \pm x^{*}, \pm x^{*}, \pm y^{*}\right)$ and their permutations, where $x^{*}, y^{*}$ satisfy Eqs.(5).

Proof: Rewriting the equations

$$
\begin{aligned}
-x^{*}+(\alpha+\beta) \tanh \left(x^{*}\right)+\beta \tanh \left(y^{*}\right) & =0 \\
-y^{*}+\alpha \tanh \left(y^{*}\right)+2 \beta \tanh \left(x^{*}\right) & =0
\end{aligned}
$$

and solving for $y^{*}$ gives

$$
y^{*}=\frac{\alpha}{\beta} x^{*}+\frac{2 \beta^{2}-\alpha \beta-\alpha^{2}}{\beta} \tanh \left(x^{*}\right) .
$$

Putting this in the first equation gives an equation for $x^{*}$ alone.

$$
F\left(x^{*}\right)=-x^{*}+(\alpha+\beta) \tanh \left(x^{*}\right)+\beta \tanh \left(\frac{\alpha}{\beta} x^{*}+\frac{2 \beta^{2}-\alpha \beta-\alpha^{2}}{\beta} \tanh \left(x^{*}\right)\right) .
$$

Defining $y(x)$ as above we can write $F(x)=-x+(\alpha+\beta) \tanh (x)+\beta \tanh (y(x))$ and find

$$
\begin{aligned}
F^{\prime}(x) & =-1+(\alpha+\beta) \operatorname{sech}^{2}(x)+\beta \operatorname{sech}^{2}(y(x)) y^{\prime}(x) \\
& =-1+(\alpha+\beta) \operatorname{sech}^{2}(x)+\beta \operatorname{sech}^{2}(y(x))\left(\frac{\alpha}{\beta}+\frac{2 \beta^{2}-\alpha \beta-\alpha^{2}}{\beta} \operatorname{sech}^{2}(x)\right) \\
& =-1+(\alpha+\beta) \operatorname{sech}^{2}(x)+\left[\alpha+\left(2 \beta^{2}-\alpha \beta-\alpha^{2}\right) \operatorname{sech}^{2}(x)\right] \operatorname{sech}^{2}(y(x)) .
\end{aligned}
$$

Note that $F(0)=0, \lim _{x \rightarrow \infty} F(x)<0$ and

$$
\begin{aligned}
F^{\prime}(0) & =-1+2 \alpha+\beta+2 \beta^{2}-\alpha \beta-\alpha^{2} \\
& =(2 \beta+\alpha-1)(\beta-\alpha+1)
\end{aligned}
$$

It is clear that $F^{\prime}(0)>0$ if $\alpha<1$ and $\beta<\alpha-1$. Since $F$ is a continuous function it follows that there is an $x^{*}>0$ such that $F\left(x^{*}\right)=0$. By symmetry $F\left(-x^{*}\right)=0$ as well.

Putting the last two theorems together with an analysis of the characteristic equation associated with the trivial solution (2) and with the results of Golubitsky et al. [1988, Chapter $\mathrm{XV} \S 4]$ leads to the following. 
Theorem 5: The trivial solution of (1) undergoes a $D_{3}$ equivariant pitchfork bifurcation along $\alpha-\beta=1$ giving rise to 12 branches of equilibria. These consist of six branches of standing wave equilibria, $\left( \pm x^{*}, \mp x^{*}, 0\right)$ and permutations, where $x^{*}$ satisfies (4), and six branches of mirror reflecting equilibria, $\left( \pm x^{*}, \pm x^{*}, \pm y^{*}\right)$ and permutations, where $x^{*}, y^{*}$ satisfy (5). The branches of standing wave equilibria are always supercritical. The branches of mirror reflecting equilibria may be sub- or supercritical.

To test our theorems, we numerically continued the branches of equilibria that arise from the pitchfork bifurcations at $\tau=1$ in Fig. 2. The results, which are shown in Fig. 4, agree with the above theorems.

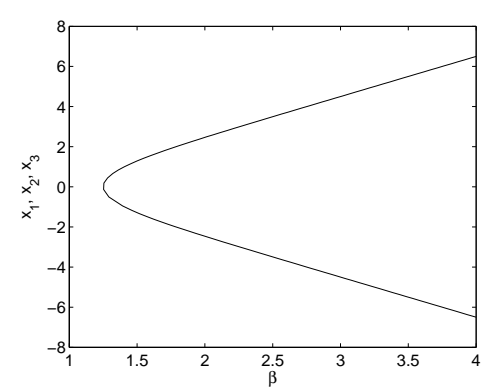

(a)

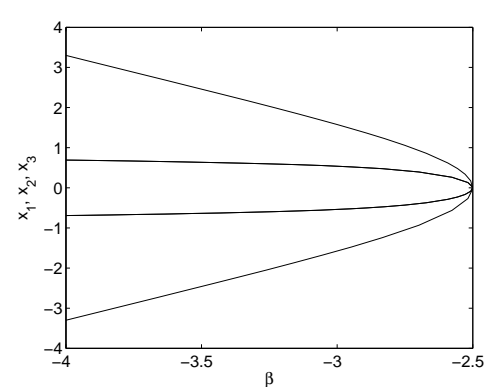

(b)

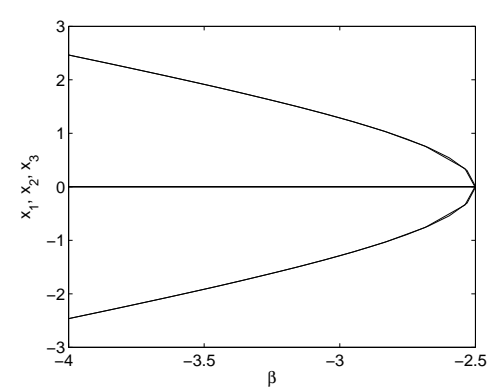

(c)

Figure 4: Illustration of (a) synchronous, (b) mirror-reflecting and (c) standing-wave equilibria arising from pitchfork bifurcations. The $x_{1}$ and $x_{2}$ curves coincide in both of the asynchronous cases.

To study further bifurcations of these equilibria, we need to consider the characteristic equation of (1) associated with each equilibrium type. To begin, we calculate the linearization about a generic nontrivial equilibrium $\left(x_{1}^{*}, x_{2}^{*}, x_{3}^{*}\right)$, viz.,

$$
\dot{\mathbf{x}}=-\mathbf{x}+A \mathbf{x}(t-\tau)
$$

where

$$
A=\left[\begin{array}{lll}
k_{1} \alpha & k_{2} \beta & k_{3} \beta \\
k_{1} \beta & k_{2} \alpha & k_{3} \beta \\
k_{1} \beta & k_{2} \beta & k_{3} \alpha
\end{array}\right]
$$


and $k_{j}=\operatorname{sech}^{2}\left(x_{j}^{*}\right)$. The corresponding characteristic equation can be found by looking for solutions of this equation of the form $\mathbf{x}=e^{\lambda t} \mathbf{u}$ where $\lambda \in \mathbb{C}$ and $\mathbf{u} \in \mathbb{C}^{3}$. Substituting this into (6) yields an equation for $\lambda$ and $\mathbf{u}=\left[u_{1}, u_{2}, u_{3}\right]^{T}$

$$
\left[\begin{array}{ccc}
-1+k_{1} \alpha e^{-\lambda \tau_{s}}-\lambda & k_{2} \beta e^{-\lambda \tau} & k_{3} \beta e^{-\lambda \tau} \\
k_{1} \beta e^{-\lambda \tau} & -1+k_{2} \alpha e^{-\lambda \tau_{s}}-\lambda & k_{3} \beta e^{-\lambda \tau} \\
k_{1} \beta e^{-\lambda \tau} & k_{2} \beta e^{-\lambda \tau} & -1+k_{3} \alpha e^{-\lambda \tau_{s}}-\lambda
\end{array}\right]\left[\begin{array}{l}
u_{1} \\
u_{2} \\
u_{3}
\end{array}\right]=0 .
$$

Requiring nontrivial solutions $(\mathbf{u} \neq 0)$ gives the characteristic equation

$$
\operatorname{det}\left[\begin{array}{ccc}
-1+k_{1} \alpha e^{-\lambda \tau_{s}}-\lambda & k_{2} \beta e^{-\lambda \tau} & k_{3} \beta e^{-\lambda \tau} \\
k_{1} \beta e^{-\lambda \tau} & -1+k_{2} \alpha e^{-\lambda \tau_{s}}-\lambda & k_{3} \beta e^{-\lambda \tau} \\
k_{1} \beta e^{-\lambda \tau} & k_{2} \beta e^{-\lambda \tau} & -1+k_{3} \alpha e^{-\lambda \tau_{s}}-\lambda
\end{array}\right]=0 .
$$

For both of the nontrivial synchronous equilibria, $\left( \pm x^{*}, \pm x^{*}, \pm x^{*}\right)$, we have $k_{j}=k=$ $\operatorname{sech}^{2}\left(x^{*}\right), j=1,2,3$, thus the characteristic equation becomes:

$$
\left(-1-\lambda+\alpha k e^{-\tau_{s} \lambda}+2 \beta k e^{-\tau \lambda}\right)\left(-1-\lambda+\alpha k e^{-\tau_{s} \lambda}-\beta k e^{-\tau \lambda}\right)^{2}=0 .
$$

Note that the form of this equation is the same as that for the trivial equilibrium (cf. Eq. (2)). Thus we expect that the nontrivial synchronous equilibria will have the same types of bifurcations as the trivial equilibrium. This leads to the following

Theorem 6: The nontrivial synchronous equilibria may undergo the following bifurcations.

1. A standard Hopf bifurcation leading to synchronous oscillations about both of the nontrivial synchronous equilibria. This bifurcation will occur when the first factor of the characteristic Eq. (8) has a pair of pure imaginary eigenvalues, if the appropriate nondegeneracy and nonresonance conditions are satisfied.

2. An equivariant Hopf bifurcation leading to 8 branches of oscillations: 3 phase-locked, 3 standing wave and 2 mirror reflecting. This bifurcation will occur when the second factor of the characteristic Eq. (8) has a pair of pure imaginary roots, if the appropriate nondegeneracy and nonresonance conditions are satisfied.

Proof: The proof of 1. is essentially the same as that of Yuan and Campbell [2004, Theorem 7.1]. The proof of 2. is essentially the same as that of Campbell et al. [2005, Theorem 4.1]. 
For the standing wave equilibria we have $k_{j}=k_{j+1}=k=\operatorname{sech}^{2}\left(x^{*}\right)$ and $k_{j+2}=1, j$ mod 3. In all cases the characteristic equation becomes:

$$
\left(\lambda+1-k \alpha e^{-\tau_{s} \lambda}+k \beta e^{-\tau \lambda}\right) P(\lambda)=0,
$$

where

$$
\begin{aligned}
P(\lambda)= & \lambda^{2}-\left((k+1) \alpha e^{-\tau_{s} \lambda}+k \beta e^{-\tau \lambda}-2\right) \lambda \\
& +k \alpha^{2} e^{-2 \tau_{s} \lambda}-2 k \beta^{2} e^{-2 \tau \lambda}+k \alpha \beta e^{-\left(\tau+\tau_{s}\right) \lambda}-(k+1) \alpha e^{-\tau_{s} \lambda}-k \beta e^{-\tau \lambda}+1 .
\end{aligned}
$$

For the mirror reflecting equilibria we have $k_{j}=k_{j+1}=k=\operatorname{sech}^{2}\left(x^{*}\right)$ and $k_{j+2}=\hat{k}=$ $\operatorname{sech}^{2}\left(y^{*}\right), j \bmod 3$. In all cases the characteristic equation becomes:

$$
\left(\lambda+1-k \alpha e^{-\tau_{s} \lambda}+k \beta e^{-\tau \lambda}\right) P(\lambda)=0,
$$

where

$$
\begin{aligned}
P(\lambda)= & \lambda^{2}-\left((k+\hat{k}) \alpha e^{-\tau_{s} \lambda}+k \beta e^{-\tau \lambda}-2\right) \lambda \\
& +k \hat{k} \alpha^{2} e^{-2 \tau_{s} \lambda}-2 k \hat{k} \beta^{2} e^{-2 \tau \lambda}+k \hat{k} \alpha \beta e^{-\left(\tau+\tau_{s}\right) \lambda}-(k+\hat{k}) \alpha e^{-\tau_{s} \lambda}-k \beta e^{-\tau \lambda}+1 .
\end{aligned}
$$

Consideration of these characteristic equations leads to the following

Theorem 7: The standing wave, respectively, mirror-reflecting, equilibria will undergo a standard Hopf bifurcation when the first factor of Eq. (9), respectively, Eq. (10), has a pair of pure imaginary roots. These bifurcations will give rise to standing wave oscillations about the respective equilibria.

Proof: The conditions of the standard Hopf bifurcation theorem for delay equations [Hale and Lunel, 1993, Section 11.1] may be easily checked.

The type of oscillation generated by such a bifurcation may be determined by considering the solutions of the linearization (6) corresponding to the pure imaginary eigenvalues associated with the bifurcation as follows. Suppose that, for a particular set of parameter 
values, the first factor of the characteristic equation (9) for the standing wave equilibria has a pair of pure imaginary roots, $\pm i \omega$. That is,

$$
\pm i \omega+1-k \alpha e^{\mp i \omega \tau_{s}}+k \beta e^{\mp i \omega \tau}=0
$$

The solutions of (6) corresponding to these roots are given by $\mathbf{x}(t)=e^{ \pm i \omega t} \mathbf{u}$ where $\mathbf{u}$ satisfies (7) with $k_{1}=k_{2}=k, k_{3}=1$, or a permutation thereof, (since we are considering the standing wave equilibria) and $\lambda= \pm i \omega$, viz.,

$$
\left[\begin{array}{ccc}
-1+k \alpha e^{\mp i \omega \tau_{s}} \mp i \omega & k \beta e^{\mp i \omega \tau} & \beta e^{\mp i \omega \tau} \\
k \beta e^{\mp i \omega \tau} & -1+k \alpha e^{\mp i \omega \tau_{s}} \mp i \omega & \beta e^{\mp i \omega \tau} \\
k \beta e^{\mp i \omega \tau} & k \beta e^{\mp i \omega \tau} & -1+\alpha e^{\mp i \omega \tau_{s}} \mp i \omega
\end{array}\right]\left[\begin{array}{l}
u_{1} \\
u_{2} \\
u_{3}
\end{array}\right]=0 .
$$

Using (11) this yields the following equations for $u_{1}, u_{2}$ and $u_{3}$

$$
\begin{aligned}
& A_{1}\left(u_{1}+u_{2}\right)+B_{2} u_{3}=0 \\
& A_{1}\left(u_{1}+u_{2}\right)+B_{3} u_{3}=0
\end{aligned}
$$

where $A_{1}=k \beta e^{\mp i \omega \tau}, B_{2}=\beta e^{\mp i \omega \tau}$ and $B_{2}=-1+\alpha e^{\mp i \omega \tau_{s}} \mp i \omega$. Clearly this yields solutions $\mathbf{u}=(u,-u, 0)$, which correspond to standing wave oscillations. Note that if we took a different permutation of the $k_{j}$ then the corresponding permutation for $\mathbf{u}$ would result. A similar calculation for the mirror reflecting equilibria yields the same result.

From the forms of (9) and (10), generically there will not be repeated pairs of pure imaginary roots of these characteristic equations. Thus we do not expect equivariant bifurcations of either the standing wave or mirror reflecting equilibria. Standard Hopf bifurcations may occur when the factor $P(\lambda)$ in either equation has a pair of pure imaginary roots, leading to different oscillation patterns than those predicted by the theorem.

\section{Numerical Results}

In this section we will use the analysis of Sec. 2 to predict what secondary bifurcations can be generated by the codimension two bifurcation points which occur in our model (1). We 
will supplement these predictions with numerical continuation studies of the model. The numerical work will focus on the parameter values of Fig. 2, i.e. $\alpha=-1.5$ and $\tau_{s}=1$.

We begin by considering the interaction between the synchronous Hopf bifurcation and the synchronous pitchfork bifurcation. Since the nonlinearities (tanh) in our model (1) are odd functions, this will be a $\mathbb{Z}_{2}$ symmetric codimension two bifurcation, which is well understood (see Guckenheimer and Holmes [1983, Section 7.5] and references therein). The main result is that there are always two bifurcation curves emanating from the codimension two point. One is a Hopf bifurcation about the nontrivial equilibrium points which creates two limit cycles simultaneously (one about each equilibrium point) and the other is a pitchfork bifurcation of these limit cycles.

More detail can be found by examining the normal form for this bifurcation [Guckenheimer and Holmes, 1983, Section 7.5]:

$$
\begin{aligned}
\dot{r} & =\mu_{1}+a_{1} r^{3}+a_{2} r z^{2} \\
\dot{\theta} & =\omega+\left(\|r, z\|^{2}\right) \\
\dot{z} & =\mu_{2}+b_{1} r^{2} z+b_{2} z^{3}
\end{aligned}
$$

where $r, \theta$ represent the amplitude and phase of the Hopf mode and $z$ the coordinate of the pitchfork mode and $\mu_{1}, \mu_{2}$ are unfolding parameters. In Guckenheimer and Holmes [1983, Section 7.5] it is shown that there are twelve different unfoldings depending on the signs of the cubic coefficients $a_{j}, b_{j}$. In our system, we know that both of the primary bifurcations (the synchronous Hopf of the trivial solution and synchronous pitchfork of the trivial solution) are supercritical bifurcations. Thus only five of these cases may occur. These five cases divide into two groups. In one group the ordering of the bifurcations is such that limit cycles around the nontrivial fixed points are unstable, and there is a region of multistability of the limit cycle about the trivial solution and the nontrivial equilibria. In the other group, the limit cycles about the nontrivial equilibria are stable.

The secondary Hopf bifurcation curves can be found numerically by continuing a curve whose initial point is a Hopf point on a branch of nontrivial equilibria. Several such curves 
for the interaction of the standard pitchfork bifurcation and standard Hopf bifurcations are shown in Fig. 5 (see Appendix A for the line style and colour convention used throughout). Points on these curves give rise to synchronous oscillations about nontrivial synchronous equilibria. If one numerically continues a branch of periodic solutions by varying $\beta$ while

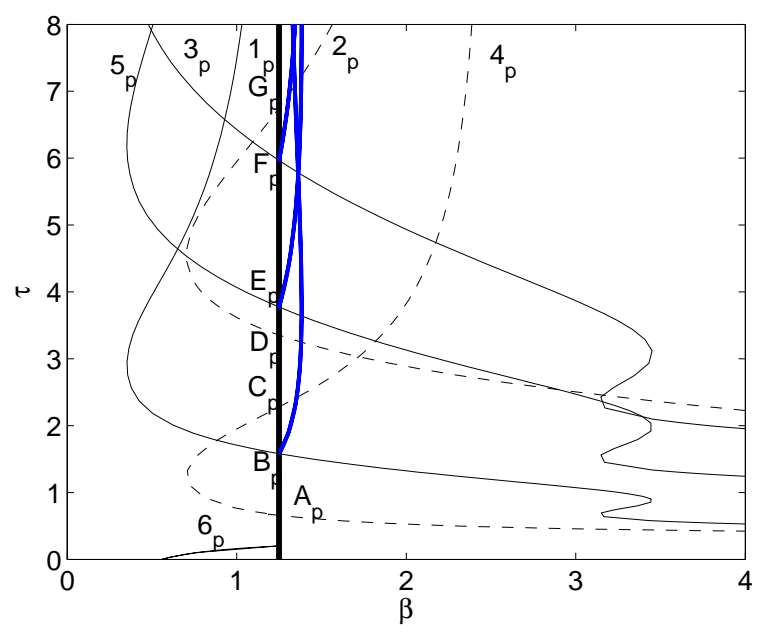

Figure 5: Illustration of secondary Hopf bifurcation curves (solid blue lines) arising from the codimension two points involving standard Hopf bifurcations and the standard pitchfork bifurcation.

keeping $\tau$ fixed, one finds that it ends in a pitchfork bifurcation of limit cycles. This is illustrated in Fig. 6. This figure shows a typical bifurcation sequence with $\tau$ held fixed near the codimension two point and $\beta$ is varied. This sequence is as follows. A standard Hopf bifurcation $(\mathrm{H})$ gives rise to a stable synchronous oscillation about the trivial solution. The resulting unstable trivial solution undergoes a standard pitchfork (PF) bifurcation to produce two branches of nontrivial synchronous equilibria, which are initially unstable. These nontrivial equilibria quickly undergo a secondary Hopf ( $\mathrm{SH}$ ) bifurcation to produce an unstable synchronous periodic solution about the nontrivial equilibria, which themselves become stable. Finally, the branch of synchronous oscillations undergoes a pitchfork of limit cycles (PFLC) bifurcation which destabilizes the synchronous oscillation originally created at the Hopf point. Note that a region of multistability between the limit cycle about zero, and the 
nontrivial equilibria can be clearly identified between the points labelled SH and PFLC in this diagram.

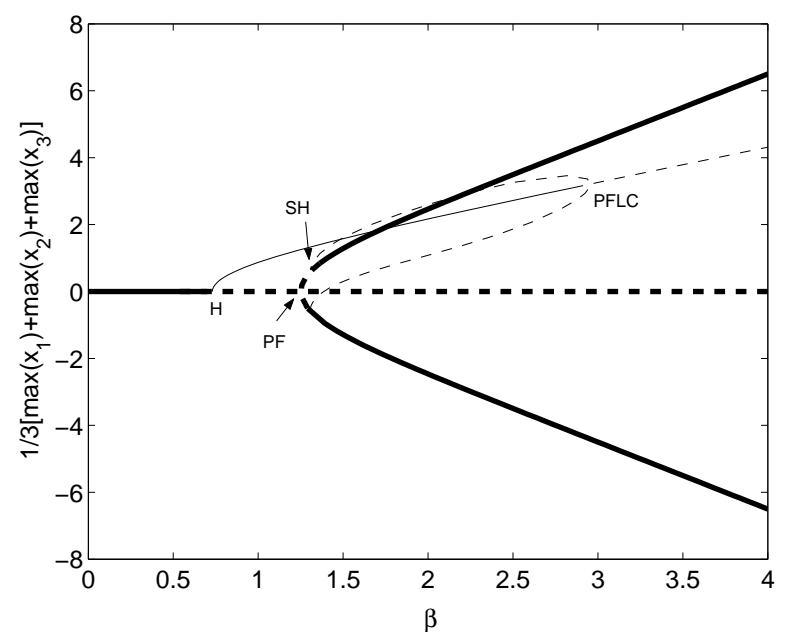

Figure 6: Illustration of numerical continuation of solution branches showing a pitchfork of limit cycle bifurcation for a branch of synchronous oscillations about a nontrivial synchronous equilibrium $(\tau=1.899)$. Solid/dashed lines denote stable/unstable solutions. Thin/thick lines denote periodic/steady state solutions.

The location of these pitchfork of limit cycle points in $\beta-\tau$ space was computed by noting where the branches of synchronous oscillations about the nontrivial equilibria turned around. These points are indicated in Fig. 7 for the interactions between standard Hopf and the standard pitchfork bifurcations. The points computed are indicated with a '*', while the curves are created by fitting splines through these points. In all cases that we studied, the arrangement of the secondary bifurcation curves is the same, and corresponds to Case Ib of Guckenheimer and Holmes [1983, Section 7.5].

Now consider the interaction between the equivariant Hopf about the trivial solution and the synchronous pitchfork bifurcation. For this case we know of no theoretical results based on normal form theory currently available. However, based on Theorem 6, we know that it is possible for the nontrivial synchronous equilibria to undergo $D_{3}$ equivariant Hopf bifurcations, leading to 8 branches of asynchronous oscillations about each nontrivial equilibrium. It is thus reasonable to conjecture that there are secondary equivariant Hopf bifurcations 


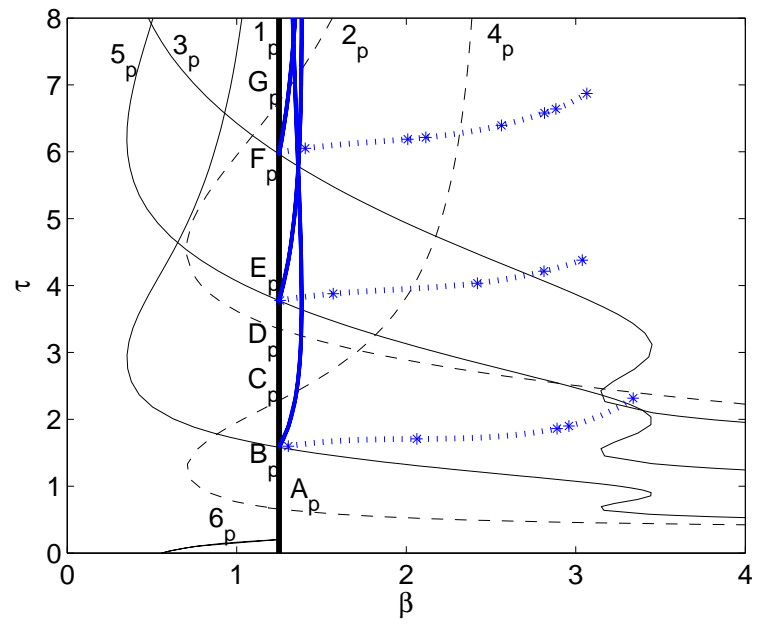

Figure 7: Illustration of PFLC bifurcation curves (dotted blue) for the codimension two points involving standard Hopf bifurcations and the standard pitchfork bifurcation.

of the nontrivial synchronous equilibria emanating from the codimension two points. Such bifurcations have been found numerically, as shown in Fig. 8. These bifurcations give rise to asynchronous oscillations about the nontrivial synchronous equilibria generated in the pitchfork bifurcation.

As in the standard Hopf / standard pitchfork case, a branch of periodic solutions generated by the secondary Hopf bifurcations emanating from an equivariant Hopf / standard pitchfork interaction ends in a pitchfork of limit cycles bifurcation, as shown in Fig. 9(a). This figure shows a typical bifurcation sequence with $\tau$ held fixed near the codimension two point and $\beta$ is varied. This sequence is as follows. An equivariant Hopf bifurcation $(\mathrm{H})$ gives rise to eight branches of asynchronous oscillation about the trivial solution: two branches of phase-locked oscillations which are initially stable, and three branches each of standing-wave and mirror-reflecting oscillations which are unstable for all $\beta$. Note that in this representation all branches corresponding to the same type of oscillation are coincident. The unstable trivial solution then undergoes a standard pitchfork (PF) bifurcation to produce two branches of nontrivial synchronous equilibria, which are initially unstable. These nontrivial equilibria quickly undergo secondary equivariant Hopf (SH) bifurcations 


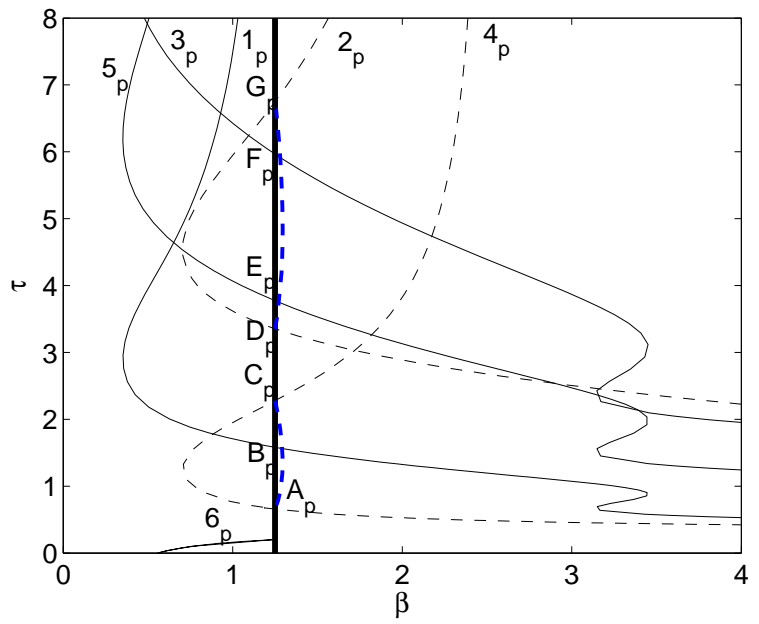

Figure 8: Illustration of secondary Hopf bifurcation curves (dashed blue) arising from codimension two points involving equivariant Hopf bifurcations and the standard pitchfork bifurcation.

to produce 16 unstable branches of asynchronous oscillations, eight about each of the nontrivial equilibria, which themselves become stable. Finally, the branches of asynchronous oscillations formed in the initial Hopf bifurcation each undergoes a pitchfork of limit cycles (PFLC) bifurcation which destabilizes the original asynchronous branch. Here we have a region of multistability between an asynchronous (phase-locked) oscillation about the trivial solution, and the nontrivial equilibria (between points $\mathrm{SH}$ and PFLC). An example of the stable solutions found in this region is shown in Fig. 9(b). Again, these PFLC points form curves in $\beta$ - $\tau$ space as shown in Fig. 10 for the three different patterns of oscillation.

For the synchronous Hopf/equivariant pitchfork interaction points, we know of no normal form results and we are unable to make any conjectures about secondary bifurcations based on the analysis of Sec. 2. Numerical results for this case reveal that secondary Hopf bifurcations do exist, as shown in Fig. 11. Two curves of standard Hopf bifurcations emanate from each codimension two point. These lead, respectively, to synchronous oscillations about the six mirror reflecting equilibria and the six standing wave equilibria. Furthermore, the branches of synchronous oscillations about nontrivial asynchronous equilibria formed by these 


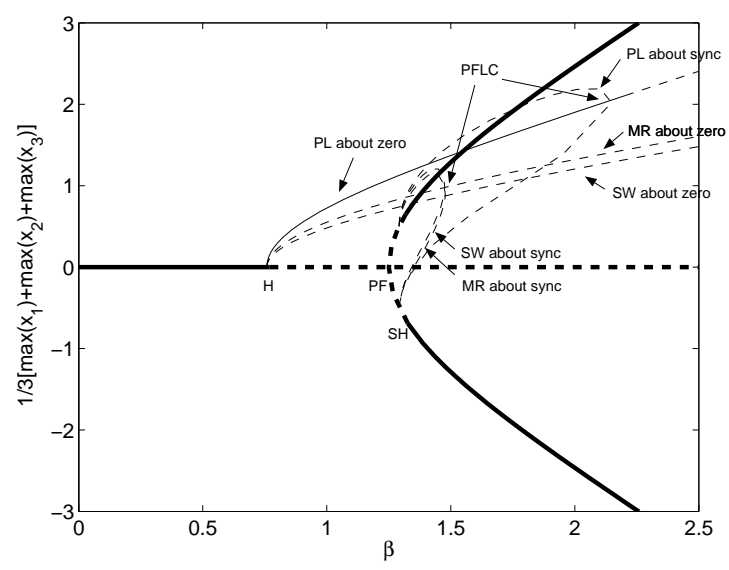

(a)

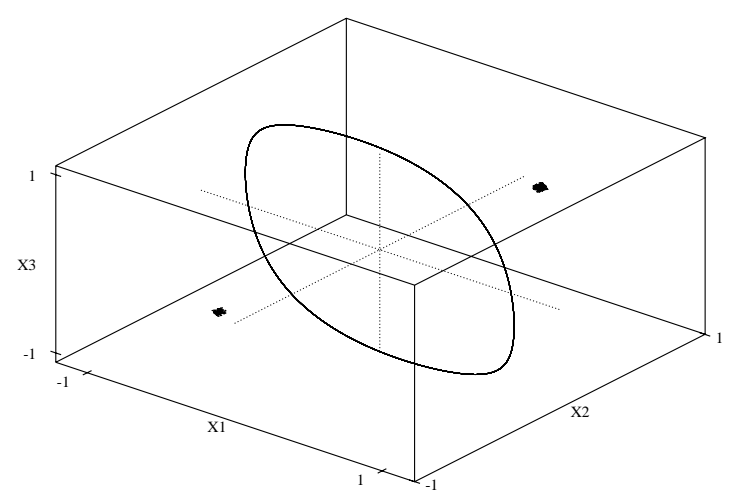

(b)

Figure 9: (a) Illustration of numerical continuation of solution branches showing pitchfork of limit cycle bifurcations for branches of asynchronous oscillations about a nontrivial synchronous equilibrium $(\tau=1.5317)$. (b) An example of multistability. At $\beta=1.3, \tau=1.8$ two nontrivial synchronous equilibrium points and a phase-locked oscillation about the trivial solution are all stable. 


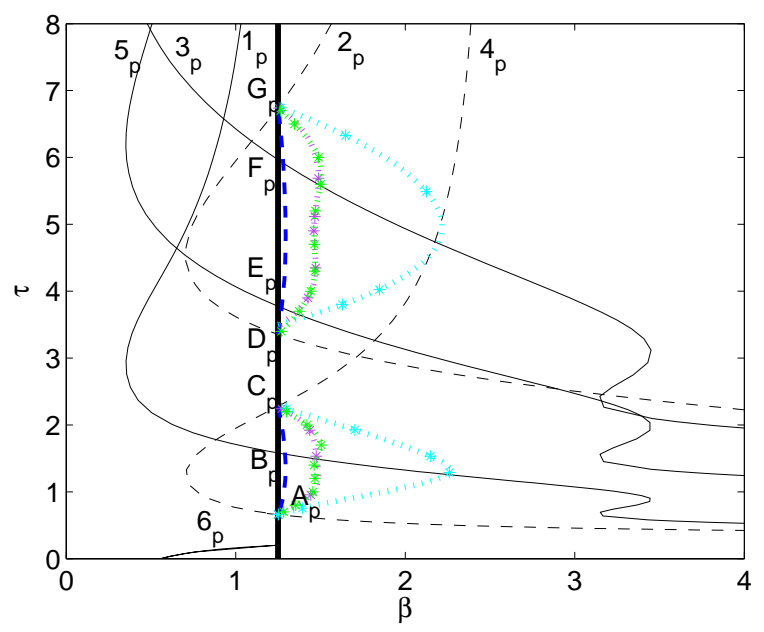

Figure 10: Illustration of PFLC bifurcation curves (dotted green, cyan and purple) for the codimension two points involving the standard pitchfork bifurcation and equivariant Hopf bifurcations.

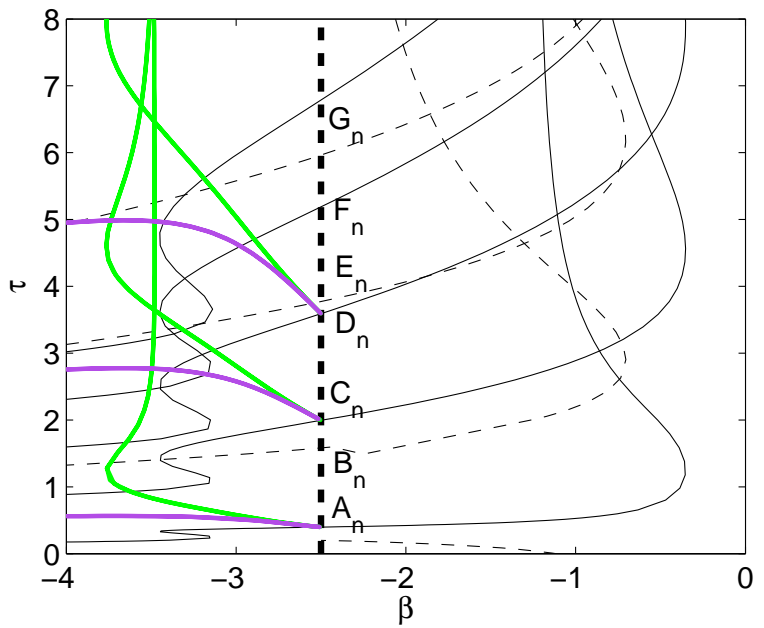

Figure 11: Illustration of secondary Hopf bifurcation curves for the codimension two points involving standard Hopf bifurcations and the equivariant pitchfork bifurcation. Two types of standard Hopf bifurcations emanate from each point: one corresponding to bifurcation of the mirror reflecting equilibria (purple) and one to bifurcation of the standing wave equilibria (green). 
secondary bifurcations end in pitchfork bifurcations of limit cycles as in the positive $\beta$ case. This is illustrated in Fig. 12. This figure shows a typical bifurcation sequence with $\tau$ held

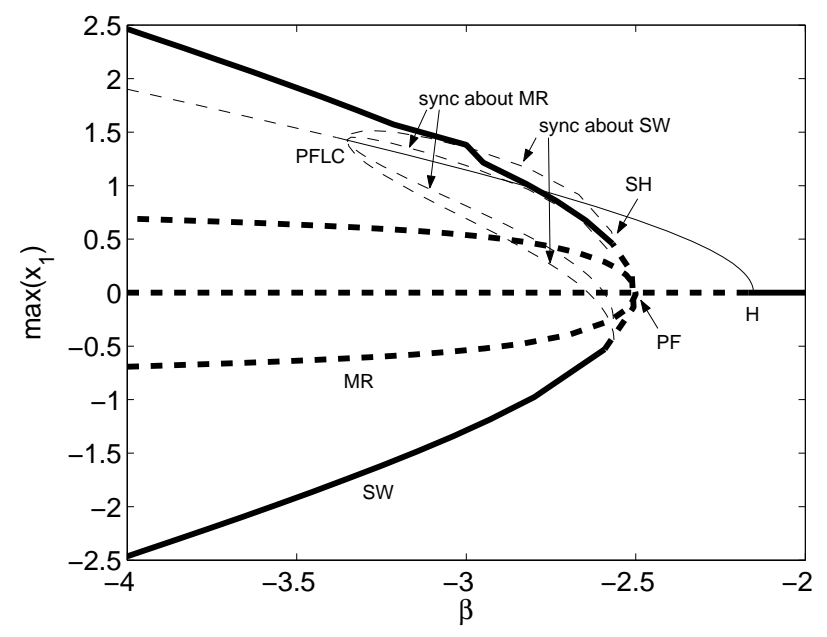

Figure 12: Illustration of numerical continuation of solution branches showing pitchfork limit cycle bifurcations for synchronous oscillations about nontrivial asynchronous equilibria $(\tau=0.41318)$.

fixed near the codimension two point and $\beta$ is varied. This sequence is as follows. A standard Hopf bifurcation $(\mathrm{H})$ gives rise to a stable synchronous oscillation about the trivial solution. The resulting unstable trivial solution undergoes an equivariant pitchfork (PF) bifurcation to produce 12 branches of nontrivial asynchronous equilibria (six each of standing-wave and mirror-reflecting type), which are initially unstable. These nontrivial equilibria quickly undergo secondary Hopf (SH) bifurcations to produce unstable synchronous periodic solutions about the nontrivial equilibria, which themselves become stable in the case of standing-wave equilibria. Finally, the branches of synchronous oscillations undergo pitchfork of limit cycle (PFLC) bifurcations which destabilize the synchronous oscillation originally created at the Hopf point. Again, we note a region of multistability, this time between the limit cycle about the trivial solution, and the standing-wave equilibria. The curves formed by the PFLC points are shown in Fig. 13.

For the equivariant Hopf/equivariant pitchfork interaction points, we know of no normal 


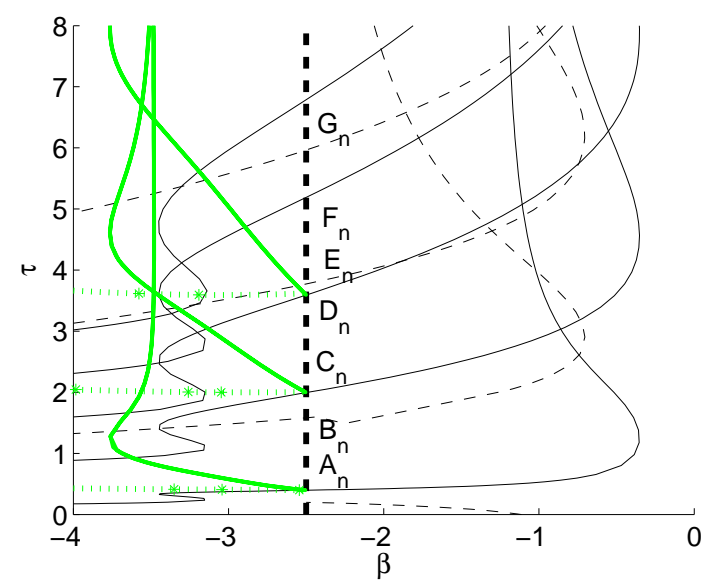

(a)

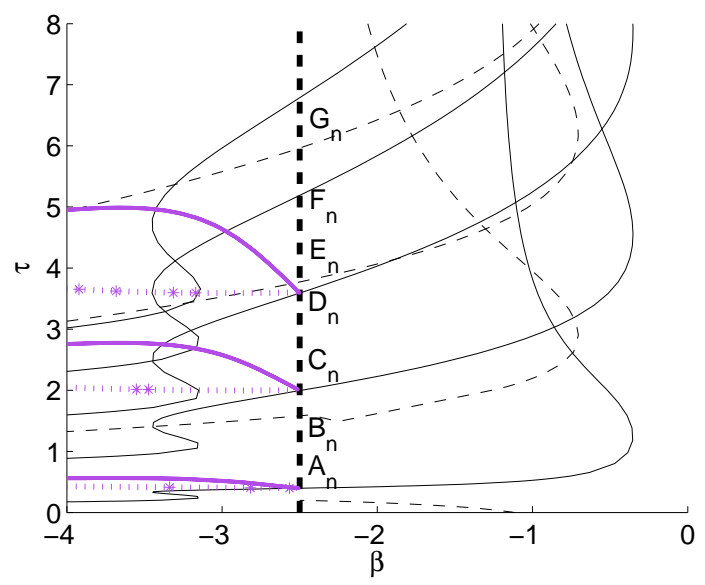

(b)

Figure 13: Illustration of PFLC bifurcation curves for codimension two points involving standard Hopf bifurcations and the equivariant pitchfork bifurcation. (a) PFLC bifurcation of synchronous oscillations about standing wave equilibria (dotted green). (b) PFLC bifurcation of synchronous oscillations about mirror reflecting equilibria (dotted purple).

form results. From Theorem 5, we predict that there are six branches of standing-wave equilibria and six branches of mirror-reflecting equilibria created by the pitchfork bifurcation, and from Theorem 7 that each branch may undergo a standard Hopf bifurcation leading to a branch of standing wave oscillations. Other standard Hopf bifurcations are also possible, but we are not able to predict their type. It is thus reasonable to conjecture that there are at least 12 curves of secondary standard Hopf bifurcations, leading to standing wave oscillations about each of asynchronous equilibria, emanating from the codimension two points. Note from the discussion of the characteristic equations (9)-(10), that all the standing wave equilibria must undergo a Hopf bifurcation at the same time and similarly for the mirror reflecting equilibria. Thus we expect that the six curves corresponding to the standing wave equilibria will be coincident and similarly for the mirror reflecting equilibria. Evidence to support this conjecture is shown in Fig. 14 where numerically computed secondary Hopf curves for equivariant Hopf / equivariant pitchfork interaction points are shown. Here, just 
two of the 12 possible curves are shown, one for standing-wave oscillations about standingwave equilibria, and one for standing-wave oscillations about mirror-reflecting equilibria. In each of these cases there are six possible curves, corresponding to the three permutations and two reflections of each of the equilibria. These curves are coincident. In addition, the figure shows curves of standard Hopf bifurcations leading to mirror-reflecting oscillations about standing wave equilibria and mirror reflecting equilibria respectively. As for the standing wave oscillations, this is two of 12 curves that occur. Note that the curves corresponding to standing wave oscillations about standing wave equilibria and mirror reflecting oscillations about mirror reflecting equilibria are coincident. Furthermore, the branches of asynchronous

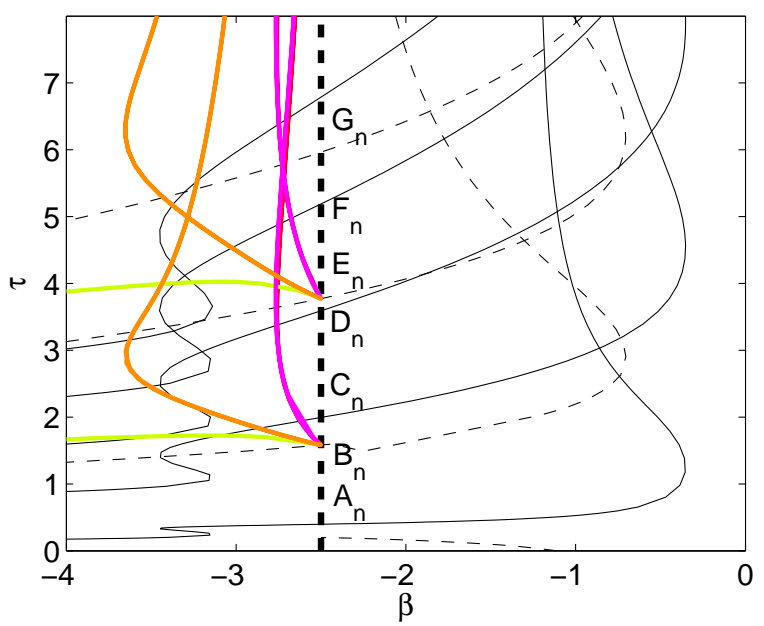

Figure 14: Illustration of secondary Hopf bifurcation curves for the codimension two points involving equivariant Hopf bifurcations and the equivariant pitchfork bifurcation. Four types curves of standard Hopf bifurcations emanate from each point: bifurcations of standing wave oscillations (magenta) and mirror reflecting oscillations (orange) about standing wave equilibria, and bifurcations of standing wave oscillations (light green) and mirror reflecting oscillations (red) about mirror reflecting equilibria.

oscillations about nontrivial asynchronous equilibria formed by these secondary bifurcations end in pitchfork of limit cycle bifurcations as in each of the above cases. This is illustrated in Fig. 15. This figure shows a typical bifurcation sequence with $\tau$ held fixed near the codimension two point and $\beta$ is varied. This sequence is as follows. An equivariant Hopf 
(H) bifurcation gives rise to an unstable asynchronous oscillation (mirror-reflecting in this case) about the trivial solution. Subsequently, the unstable trivial solution undergoes an equivariant pitchfork $(\mathrm{PF})$ bifurcation to produce 12 branches of nontrivial asynchronous equilibria (standing-wave shown), which are initially unstable. These nontrivial equilibria undergo secondary equivariant Hopf (SH) bifurcations to produce unstable asynchronous periodic solutions about the nontrivial equilibria (MR about SW shown). Here, the branch of mirror-reflecting oscillations about the standing-wave equilibrium is subcritical and ends in a pitchfork of limit cycles bifurcation. PFLC points for equivariant Hopf, equivariant

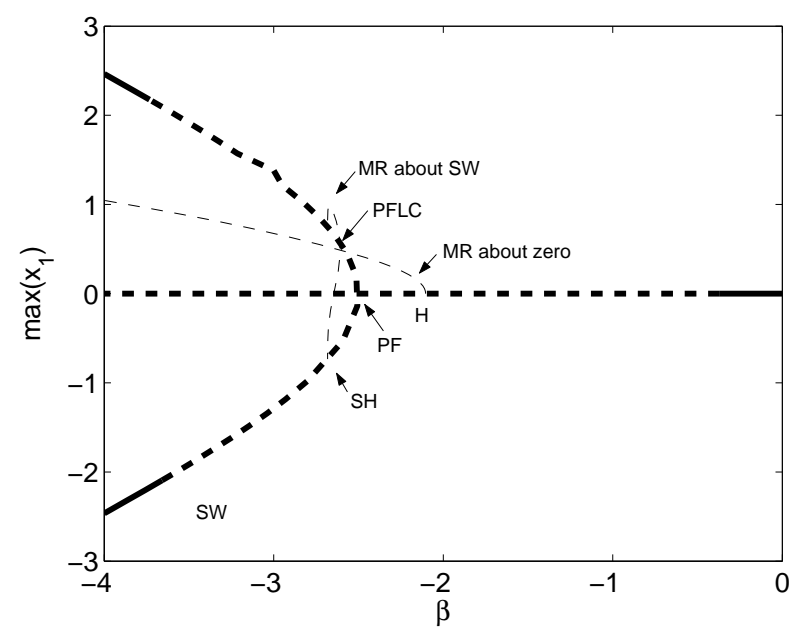

Figure 15: Illustration of numerical continuation of solution branches showing a pitchfork of limit cycles bifurcation for a branch of mirror-reflecting oscillations about a standing-wave equilibrium $(\tau=4)$. Note that the trivial solution became unstable in a standard Hopf bifurcation near $\beta=-0.5$.

pitchfork interactions are shown in Fig. 16. Again, the PFLC curves are created by fitting splines through these points. In the case of standing-wave oscillations about mirror-reflecting equilibria (light green curves), the spline was fit to the PFLC points as well as the maximum point of the secondary Hopf curves since it was found that periodic solution branches continued from points beyond this extremum were supercritical (unlike those prior to the extremum), and did not terminate in a PFLC bifurcation.

We close this section by noting that the secondary Hopf bifurcations emanating from the 


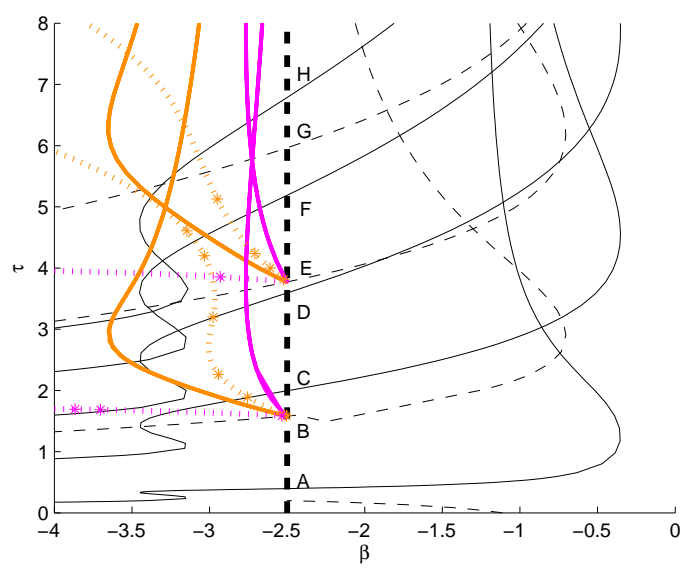

(a)

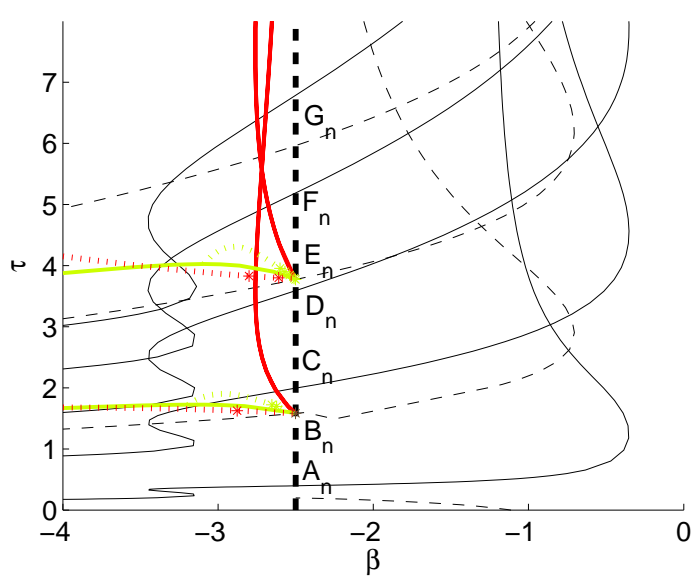

(b)

Figure 16: Illustration of PFLC bifurcation curves for the codimension two points involving equivariant Hopf bifurcations and the equivariant pitchfork bifurcation. (a) PFLC bifurcation of asynchronous oscillations about standing wave equilibria (dotted magenta and orange). (b) PFLC bifurcation of asynchronous oscillations about mirror reflecting equilibria (dotted red and light green). 
various codimension two bifurcation points in our model (1) lead to many different patterns of oscillation. Some examples are shown in Fig. 17. Of these, we have only found parameter values where two of these are stable: the synchronous oscillations about the trivial solution and the standing wave oscillations about the trivial solution. As discussed above, these patterns are a result of the many interactions of the standard and equivariant steady state (pitchfork) bifurcations with the standard and equivariant Hopf bifurcations exhibited by the model.

\section{Discussion}

We have studied, via numerical continuation, the 4 types of codimension two Hopf-pitchfork interactions exhibited by (1). In the case where both bifurcations are standard, our numerical results agree qualitatively with the predictions of normal form theory. There are two secondary bifurcation branches emanating from each codimension two point: a Hopf bifurcation of limit cycles surrounding the nontrivial equilibria and a pitchfork bifurcation of these limit cycles. This agreement could be checked quantitatively by using a centre manifold reduction of the delay differential equation as was done for a standard Hopf pitchfork inter-

action in Bélair et al. [1996]. In the remaining three cases, one or more of the bifurcations is equivariant. We know of no normal form results for these situations, thus we summarize our results with some conjectures about what normal form analysis may tell us. Although we considered a ring of three oscillators, based on our analysis of the bifurcations of the trivial solution [Yuan and Campbell, 2004; Campbell et al., 2005] we know that all the codimension two points observed here are also observed in the case of $n$ oscillators. We thus state our conjectures for $D_{n}$ symmetric systems.

Conjecture 8: Consider a codimension two bifurcation point involving a standard pitchfork bifurcation and a $D_{n}$ equivariant Hopf bifurcation of an equilibrium point. There will be a secondary equivariant Hopf bifurcation emanating from the codimension two point, giving rise to $2 n+2$ branches of periodic orbits ( $n$ standing wave oscillations, $n$ mirror reflecting 

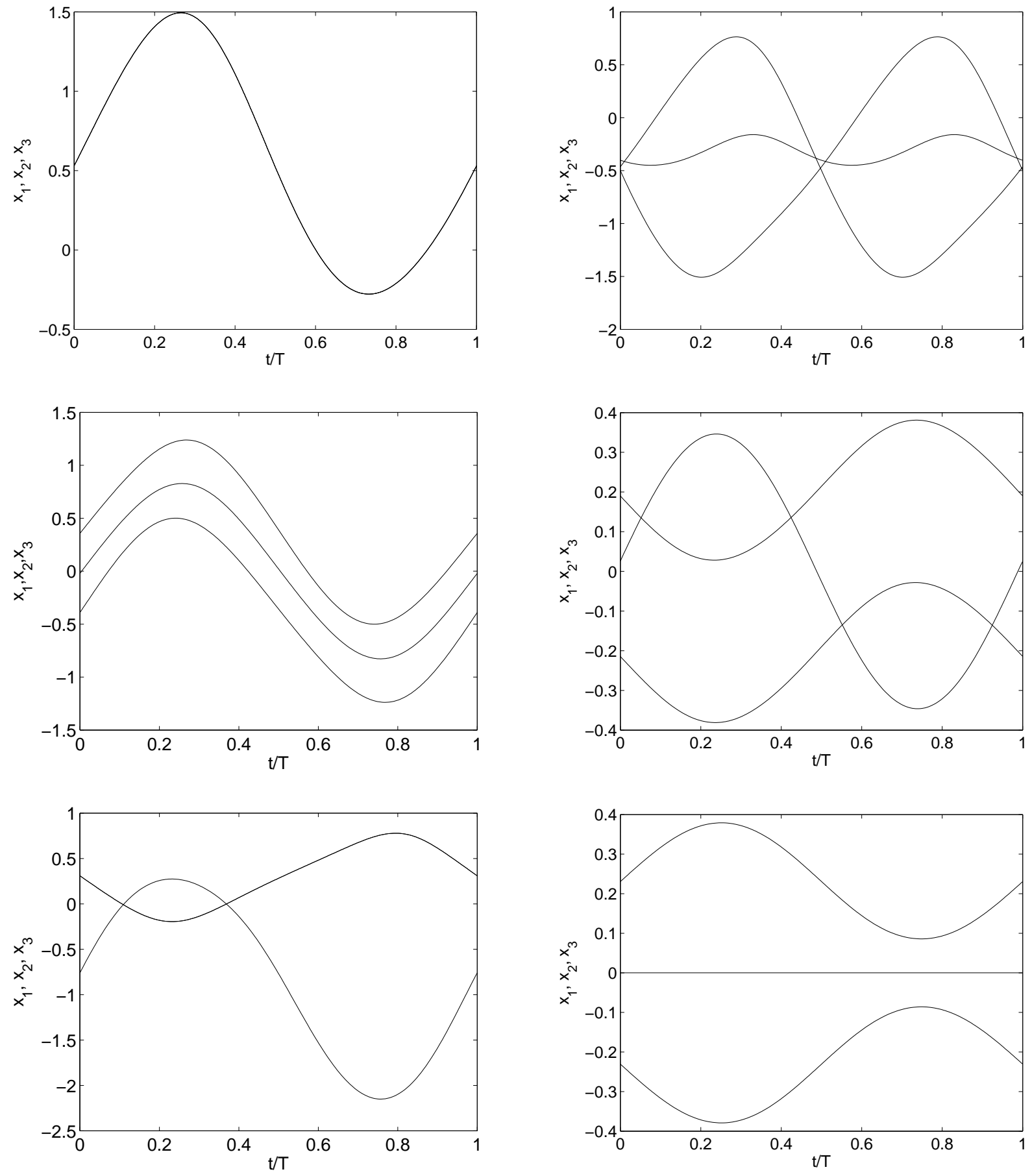

Figure 17: Illustration of the various patterns of oscillation about nontrivial equilibria exhibited by (1). From top left clockwise: synchronous oscillation about a synchronous equilibrium, standing-wave oscillation about a synchronous equilibrium, mirror-reflecting oscillation about a standing-wave equilibrium, standing-wave oscillation about a standing-wave equilibrium, mirror-reflecting oscillation about a mirror-reflecting equilibrium, synchronous oscillation about a standing-wave equilibrium. 
oscillations and 2 phase-locked oscillations) about each equilibria produced by the pitchfork bifurcation. There will also be $2 n+2$ pitchfork bifurcations of limit cycles emanating from the codimension two point.

Conjecture 9: Consider a codimension two bifurcation point involving a $D_{n}$ equivariant pitchfork bifurcation and a standard Hopf bifurcation of an equilibrium point. Note that the pitchfork bifurcation gives rise to $2 n$ standing wave equilibria and $2 n$ mirror reflecting equilibria. There will be $4 n$ secondary standard Hopf bifurcations emanating from the codimension two point, giving rise to $4 n$ synchronous periodic orbits, one about each of the $4 n$ asynchronous equilibria. There will also be $2 n$ pitchfork bifurcations of limit cycles emanating from the codimension two point.

Conjecture 10: Consider a codimension two bifurcation point involving a $D_{n}$ equivariant pitchfork bifurcation and a $D_{n}$ equivariant Hopf bifurcation of an equilibrium point. There will be $8 n$ secondary standard Hopf bifurcations emanating from the codimension two point, giving rise to $8 n$ branches of periodic orbits, two about each of the $4 n$ equilibria produced by the equivariant pitchfork bifurcation. There will also be $4 n$ pitchfork bifurcations of limit cycles.

Although we studied a specific type of oscillator in our ring, we believe that these general results should carry over to other symmetric rings of type II oscillators (i.e. the inherent oscillations are induced by a supercritical Hopf bifurcation) with time delayed coupling of the form we use here. Bifurcation diagrams similar to those we obtain are found in Burić et al. [2005] and Burić and Todorovic [2003] when studying Fitzhugh-Nagumo oscillators and in Campbell et al. [2004] when studying simple loop oscillators with time delayed coupling.

Returning to the neural system, it would seem that the choice of oscillator model we made makes the synchronous oscillations about the trivial solution fairly robust. The many other types of oscillations possible in this network are, for the most part, unstable. This might be a desirable property for a network which needs to reliably produce a particular oscillation for a range of parameters. As indicated above, we believe that using other type II 
oscillator models will give similar results, namely the secondary bifurcations will be the same. However, the locations and criticality of these secondary bifurcations may well change. In particular, it may be possible to produce systems where the secondary bifurcations produce stable oscillations of different patterns. This would lead to a network which has flexibility: changes to parameters can cause a large change in the response of the system.

\section{References}

Baldi, P. and Atiya, A. F. (1994). How delays affect neural dynamics and learning. IEEE Trans. Neural Networks, 5(4):612-621.

Bélair, J. and Campbell, S. A. (1994). Stability and bifurcations of equilibria in a multipledelayed differential equation. SIAM J. Appl. Math., 54(5):1402-1424.

Bélair, J., Campbell, S. A., and van den Driessche, P. (1996). Frustration, stability and delay-induced oscillations in a neural network model. SIAM J. Appl. Math., 56:245-255.

Burić, N., Grozdanović, I., and Vasović, N. (2005). Type I vs. type II excitable systems with delayed coupling. Chaos Solitons Fractals, 23:1221-1233.

Burić, N. and Todorovic, D. (2003). Dynamics of Fitzhugh-Nagumo excitable systems with delayed coupling. Phys. Rev. E, 67:066222.

Campbell, S. A. (1999). Stability and bifurcation of a simple neural network with multiple time delays. In Ruan, S., Wolkowicz, G. S. K., and Wu, J., editors, Differential equations with application to biology, Fields Institute Communications, volume 21, pages 65-79. AMS.

Campbell, S. A., Edwards, R., and van den Driessche, P. (2004). Delayed coupling between two neural network loops. SIAM J. Appl. Math., 65(1):316-335.

Campbell, S. A., Ncube, I., and Wu, J. (2006). Multistability and stable asynchronous periodic oscillations in a multiple-delayed neural system. Phys. D, 214(2):101-119. 
Campbell, S. A., Ruan, S., and Wei, J. (1999). Qualitative analysis of a neural network model with multiple time delays. Internat. J. Bifur. Chaos, 9(8):1585-1595.

Campbell, S. A., Yuan, Y., and Bungay, S. D. (2005). Equivariant Hopf bifurcation in a ring of identical cells with delayed coupling. Nonlinearity, 18:2827-2846.

Cohen, M. and Grossberg, S. (1983). Absolute stability of global pattern formation and parallel memory storage by competitive neural networks. IEEE Trans. Systems Man Cybernet., 13(5):815-826.

Dhooge, A., Govaerts, W., and Kuznetsov, Y. A. (2003). Matcont: A matlab package for numerical bifurcation analysis of ODEs. ACM Trans. Math. Soft., 29:141-164. www.matcont.ugent.be.

Doedel, E. J., Keller, H. B., and Kernevez, J. P. (1991a). Numerical analysis and control of bifurcation problems, (I) Bifurcation in finite dimensions. Internat. J. Bifur. Chaos, $1(3): 493-520$.

Doedel, E. J., Keller, H. B., and Kernevez, J. P. (1991b). Numerical analysis and control of bifurcation problems, (II) Bifurcation in infinite dimensions. Internat. J. Bifur. Chaos, $1(4): 745-772$.

Engelborghs, K., Luzyanina, T., and Samaey, G. (2001). DDE-BIFTOOL v. 2.00: a Matlab package for bifurcation analysis of delay differential equations. Technical Report TW-330, Department of Computer Science, K. U. Leuven, Leuven, Belgium.

Ermentrout, B. (2002). Simulating, analyzing, and animating dynamical systems: A guide to XPPAUT for researchers and students. SIAM, Philadelphia.

Golubitsky, M., Stewart, I., and Schaeffer, D. G. (1988). Singularities and groups in bifurcation theory, volume 2. Springer Verlag, New York.

Grossberg, S. (1978). Competition, decision, and consensus. J. Math. Anal. Appl., 66(2):470493. 
Grossberg, S. (1980). Biological competition: Decision rules, pattern formation, and oscillations. Proc. Nat. Acad. Sci. USA, 77(4):2338-2342.

Guckenheimer, J. and Holmes, P. J. (1983). Nonlinear oscillations, dynamical systems and bifurcations of vector fields. Springer-Verlag, New York.

Guo, S. (2005). Spatio-temporal patterns of nonlinear oscillations in an excitatory ring network with delay. Nonlinearity, 18:2391-2407.

Guo, S. and Huang, L. (2003). Hopf bifurcating periodic orbits in a ring of neurons with delays. Phys. D, 183:19-44.

Guo, S. and Huang, L. (2005). Global continuation of nonlinear waves in a ring of neurons. Proc. Roy. Soc. Edinburgh, 135A:999-1015.

Guo, S. and Huang, L. (2006). Nonlinear waves in a ring of neurons with delays. IMA J. Appl. Math. (to appear).

Guo, S., Huang, L., and Wang, L. (2004). Linear stability and Hopf bifurcation in a two neuron network with three delays. Internat. J. Bifur. Chaos, 14:2799-2810.

Hale, J. K. and Lunel, V. (1993). Introduction to functional differential equations. Springer Verlag, New York.

Hopfield, J. J. (1982). Neural networks and physical systems with emergent collective computational abilities. Proc. Nat. Acad. Sci. USA, 79(8):2554-2558.

Hopfield, J. J. (1984). Neurons with graded response have collective computational properties like those of two-state neurons. Proc. Nat. Acad. Sci. USA, 81:3088-3092.

Krawcewicz, W., Vivi, P., and Wu, J. (1997). Computation formulae of an equivariant degree with applications to symmetric bifurcations. Nonlinear Stud., 4(1):89-119. 
Krawcewicz, W. and Wu, J. (1999). Theory and applications of Hopf bifurcations in symmetric functional-differential equations. Nonlinear Anal., 35(7, Series A: Theory Methods):845-870.

Kuznetsov, Y. A. (1995). Elements of applied bifurcation theory. Springer-Verlag, Berlin/New York.

Marcus, C. M., Waugh, F. R., and Westervelt, R. M. (1991). Nonlinear dynamics and stability of analog neural networks. Phys. D, 51:234-247.

Marcus, C. M. and Westervelt, R. M. (1989). Stability of analog neural networks with delay. Phys. Rev. A, 39(1):347-359.

Ncube, I., Campbell, S. A., and Wu, J. (2003). Change in criticality of synchronous Hopf bifurcation in a multiple-delayed neural system. Fields Inst. Commun., 36:17-193.

Orosz, G., Krauskopf, B., and Wilson, R. E. (2005). Bifurcations and multiple traffic jams in a car-following model with reaction-time delay. Phys. D, 211(3):277-293.

Orosz, G. and Stépán, G. (2004). Hopf bifurcation calculations in delayed systems with translational symmetry. J. Nonlinear Sci., 14(6):505-528.

Orosz, G. and Stépán, G. (2006). Subcritical Hopf bifurcations in a car-following model with reaction-time delay. Proc. Roy. Soc. London A. (to appear).

Orosz, G., Wilson, R. E., and Krauskopf, B. (2004). Global bifurcation investigation of an optimal velocity traffic model with driver reaction time. Phys. Rev. E, 70(2):026207.

Shayer, L. P. and Campbell, S. A. (2000). Stability, bifurcation and multistability in a system of two coupled neurons with multiple time delays. SIAM J. Appl. Math., 61(2):673-700.

Wu, J. (1998). Symmetric functional-differential equations and neural networks with memory. Trans. Amer. Math. Soc., 350(12):4799-4838. 
Wu, J., Faria, T., , and Huang, Y. S. (1999). Synchronization and stable phase-locking in a network of neurons with memory. Math. Comput. Modelling, 30(1-2):117-138.

Yuan, Y. and Campbell, S. A. (2004). Stability and synchronization of a ring of identical cells with delayed coupling. J. Dynam. Differential Equations, 16(1):709-744. 


\section{A Line Style and Colour Convention}

\begin{tabular}{||l|c|c|c||}
\hline \multicolumn{3}{|c||}{ Primary bifurcations } \\
\hline Bifurcation / Oscillation & Equilibrium & Line style & Colour \\
\hline standard Hopf & trivial & solid & black \\
equivariant Hopf & trivial & dashed & black \\
standard pitchfork & trivial & solid (wide) & black \\
equivariant pitchfork & trivial & dashed (wide) & black \\
\hline \hline \multicolumn{2}{|c|}{ Secondary bifurcations } \\
\hline Bifurcation / Oscillation & Equilibrium & Line style & Colour \\
\hline standard Hopf / sync & sync & solid & blue \\
equivariant Hopf / PL,SW,MR & sync & dashed & blue \\
standard Hopf / sync & SW & solid & green \\
standard Hopf / sync & MR & solid & purple \\
standard Hopf / SW & SW & solid & magenta \\
standard Hopf / SW & MR & solid & light green \\
standard Hopf / MR & SW & solid & orange \\
standard Hopf / MR & MR & solid & red \\
PFLC / sync & sync & dotted & blue \\
PFLC / MR & sync & dotted & purple \\
PFLC / SW & sync & dotted & green \\
PFLC / PL & sync & dotted & cyan \\
\hline
\end{tabular}

Table 1: The line style and colour convention used for the bifurcation curves. The line style is determined by the type of bifurcation, while the line colour is determined by a combination of the type of oscillation and the type of equilibrium about which the oscillations are produced. 